\title{
Vertically-Suspended Environmental Enrichment Alters the Velocity Profiles of Circular Fish Rearing Tanks
}

\author{
Alissa M. Muggli1 ${ }^{1}$ Joseph M. Barnes ${ }^{1}$, Michael E. Barnes ${ }^{2 *}$ \\ ${ }^{1}$ School of Engineering, Benedictine College, Atchison, KS, USA \\ ${ }^{2}$ South Dakota Department of Game, Fish, and Parks, McNenny State Fish Hatchery, Spearfish, SD, USA \\ Email: *mike.barnes@state.sd.us
}

How to cite this paper: Muggli, A.M., Barnes, J.M. and Barnes, M.E. (2019) Vertically-Suspended Environmental Enrichment Alters the Velocity Profiles of Circular Fish Rearing Tanks. World Journal of Engineering and Technology, 7, 208-226. https://doi.org/10.4236/wjet.2019.71014

Received: January 15, 2019

Accepted: February 11, 2019

Published: February 14, 2019

Copyright $\odot 2019$ by author(s) and Scientific Research Publishing Inc. This work is licensed under the Creative Commons Attribution International License (CC BY 4.0).

http://creativecommons.org/licenses/by/4.0/

\begin{abstract}
The inclusion of vertically-suspended environmental enrichment in circular tanks has produced substantial benefits during fish rearing. This study examined the tank water velocity profiles of four different vertically-suspended structures (rod array, extended rod array, single angle array, double angle array) and a control (no environmental enrichment) at two incoming water velocities $(18.3 \mathrm{~cm} / \mathrm{s}$ and $54.9 \mathrm{~cm} / \mathrm{s})$ in $1.8-\mathrm{m}$ diameter circular tanks. At both of the incoming water velocities, overall water velocities throughout the tank were significantly reduced with the addition of any environmental enrichment in comparison to the control. In addition, the overall water velocities in the double angle array were significantly lower than the other three enrichment treatments. The pattern of significant reductions in velocity with the use of any environmental enrichment, with further significant velocity reductions in the double angle treatment, was repeated when the data were combined for each sampling depth, radius from the center, and degree (circular arc). Although considerable variation in water velocity was observed at each specific sampling location with both incoming velocities, significantly lower velocities were observed at nearly every sampling location with the addition of any environmental enrichment to the circular tank. In addition, the double angle array consistently produced the lowest velocities among the environmental enrichment treatments. The changes in velocity profiles from vertically-suspended environmental enrichment may be at least partially-responsible for the frequently-observed improvements in fish growth.
\end{abstract}

\section{Keywords}

Environmental Enrichment, Vertically-Suspended Structure, Circular Tank, Water Velocity 


\section{Introduction}

The ideal fish rearing tank would have uniform water quality, be self-cleaning, provide water velocity for fish exercise, and efficiently use the available floor plan. It is also important that all of the fish in the tank contact flowing water [1]. Circular tanks excel at homogeneously distributing dissolved oxygen, and eliminating biosolids [2] [3] [4], as well as providing adequate velocity for exercise [5] [6] [7] [8]. However, they cannot share tank sidewalls, precluding optimum space management [9]. Despite these floor plan limitations, circular tanks are commonly used in production aquaculture [10].

Water typically enters a circular tank through a tangential inlet, such as a spray bar, and exits through a screened central bottom outlet [4]. Compared to angular tanks, circular tanks have higher velocities and more stable flow patterns, resulting in self-cleaning and improved water quality benefits [2] [4] [11]. Several studies have generally described flow patterns in circular tanks [1] [2] [4] [12] [13] [14]. Circular tank velocity profiles are primarily affected by the size of the tank, the geometry of the water inlet structure, the number and location of outlet structures, the number and size of fish in the tank, and the flow and velocity of the incoming water [4] [9] [12] [13] [14]. Flow patterns are also significantly affected by the addition of environmental enrichment structures to the tanks [10].

Environmental enrichment is the placement of material or structure within otherwise-sterile hatchery rearing units to try and imitate the natural environment [15]. Some enrichment techniques include placing woody or stony debris, plant or root material, plastic plants, or concrete blocks directly into the rearing tank [16]-[23]. However, placing structures within a circular tank can be problematic because they can interfere with hydraulic self-cleaning, increasing labor requirements and the risk of disease from trapped food and feces [2] [4] [12] [23] [24] [25] [26]. In order to avert these hazards, an environmental enrichment technique using vertically-suspended aluminum rods that did not affect the hydraulic self-cleaning of circular tanks was created [27]. These vertically-suspended structures were shown to significantly improve rainbow trout (Oncorhynchus mykiss) rearing performance [27]. Subsequent investigations evaluating various types of vertically-suspended structures, including strings of spheres, plastic pipes, and aluminum angles, have also indicated positive effects during the rearing of multiple salmonid species [28] [29] [30] [31].

Little research has been published describing the flow profile of a circular tank with vertically-suspended environmental enrichment. The lone published study used only one type of suspended structure (a small aluminum rod array), and two different incoming water flow rates, but did not describe the actual water velocities [10]. Given that incoming water velocities influence circular tank flow patterns [4], and the positive effects observed when using velocity manipulation to exercise fish during hatchery rearing [5] [6] [7] [8] [32] [33] [34], there is a need to evaluate the flow dynamics of circular tanks containing suspended 
enrichment at different incoming water velocities. In addition, there is a need to describe the impacts of circular tank flow patterns for a variety of suspended structures beyond just the small array used previously [10]. As such, the objective of this study was to describe the flow patterns of circular tanks containing a number of different vertically-suspended enrichment structures at two different water velocities.

\section{Materials and Methods}

All measurements were recorded in a $1.82-\mathrm{m}$ diameter and $0.8-\mathrm{m}$ deep fiberglass circular tank fitted with a central drain and a spray bar at McNenny State Fish Hatchery, rural Spearfish, South Dakota, USA and filled with water to a depth of $0.585 \mathrm{~m}$ (Figure 1). The incoming flow rate of water through the spray bar was set at $22 \mathrm{~L} / \mathrm{min}$ throughout the experiment. Water entered from the spray bar, rotated in a counterclockwise direction, and exited the tank via the drain screen. The spray bar was adjusted to create two water velocities, $18.3 \mathrm{~cm} / \mathrm{s}$ and $54.9 \mathrm{~cm} / \mathrm{s}$. Velocity measurements were taken with a FP111 Flow Probe (Global Water Instrumentation, College Station, Texas, USA).

In addition to the two velocities, four types of environmental enrichment (rod array, extended rod array, single angle array, double angle array), along with a control scenario with no enrichment, were included in this study. The structures used are described in Table 1 and illustrated in Figure 2(a)-(d). The rods were made of aluminum ( $1.0 \mathrm{~cm}$ diameter, $57.2 \mathrm{~cm}$ length) as were the angles $(2.5 \mathrm{~cm}$ width on each side, $55.9 \mathrm{~cm}$ long). The rods or angles were inserted into rectangles of corrugated plastic suspended above the surface of the water. Figure 3 (a)-(c) shows close-up views of the rod or angle arrays used, while Figure 4(a) \& Figure 4(b) show individual angle dimensions.

The locations of the velocity measurements were determined by imposing a grid system across the top of the tank, forming equal quadrants. Each radial

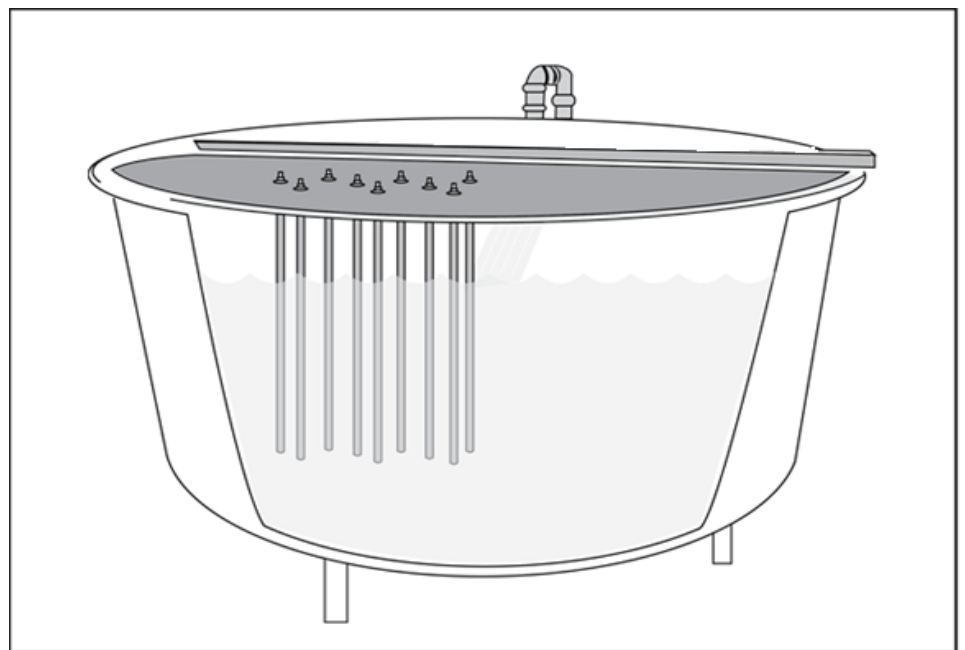

Figure 1. Schematic of circular tank, shown with nine vertical rods suspended from a tank cover. 
Table 1. Environmental enrichment structures used during an evaluation of flow dynamics in a 1.8-meter diameter circular tank.

\begin{tabular}{cccc}
\hline Structure & Description & Source & Figure \\
\hline Rod array & Nine rods equally spaced in a $27.3 \mathrm{~cm}$ by $47.0 \mathrm{~cm}$ rectangle. & Kientz and Barnes 2016 & $3(\mathrm{a})$ \\
Extended rod array & Fifteen rods evenly spaced in a $43.2 \mathrm{~cm}$ by $54.6 \mathrm{~cm}$ rectangle. & Barnes, pers. comm. & $3(\mathrm{~b})$ \\
Single angle array & Four angles evenly spaced in a $40.6 \mathrm{~cm}$ by $32.4 \mathrm{~cm}$ rectangle. & Krebs et al. 2018 & $3(\mathrm{c})$ \\
Double angle array & Two aluminum angle arrays (described previously). & Krebs et al. 2018 & $3(\mathrm{c}), 5$ \\
\hline
\end{tabular}

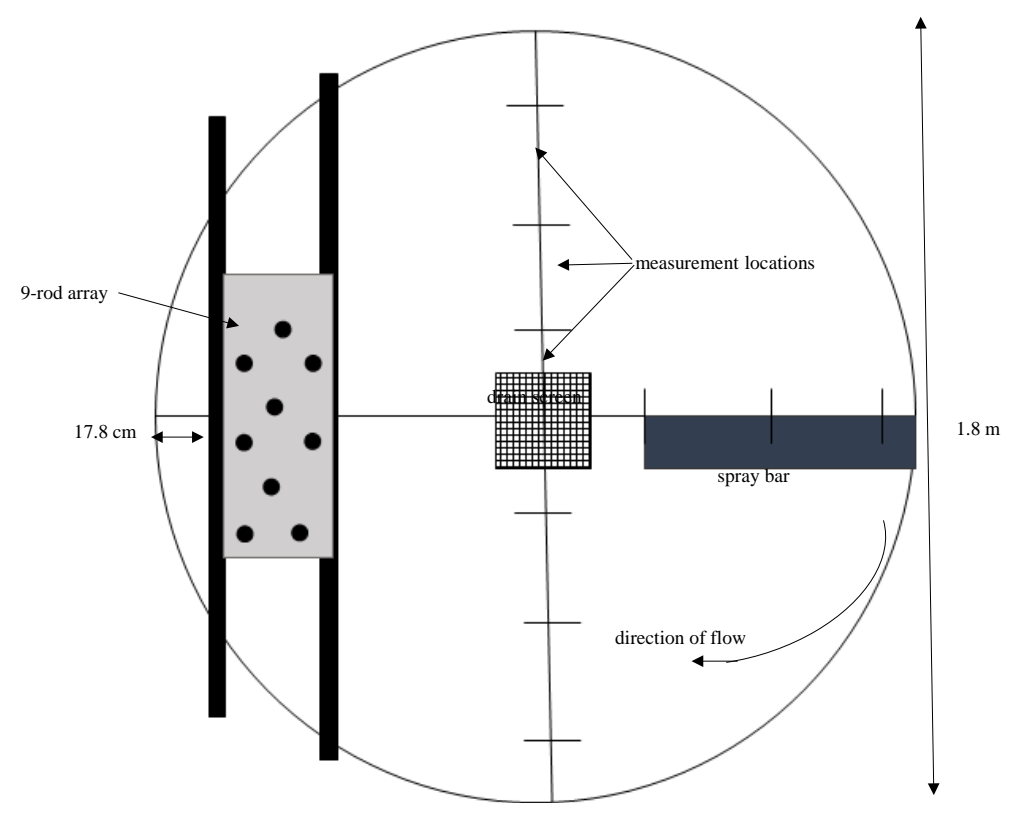

(a)

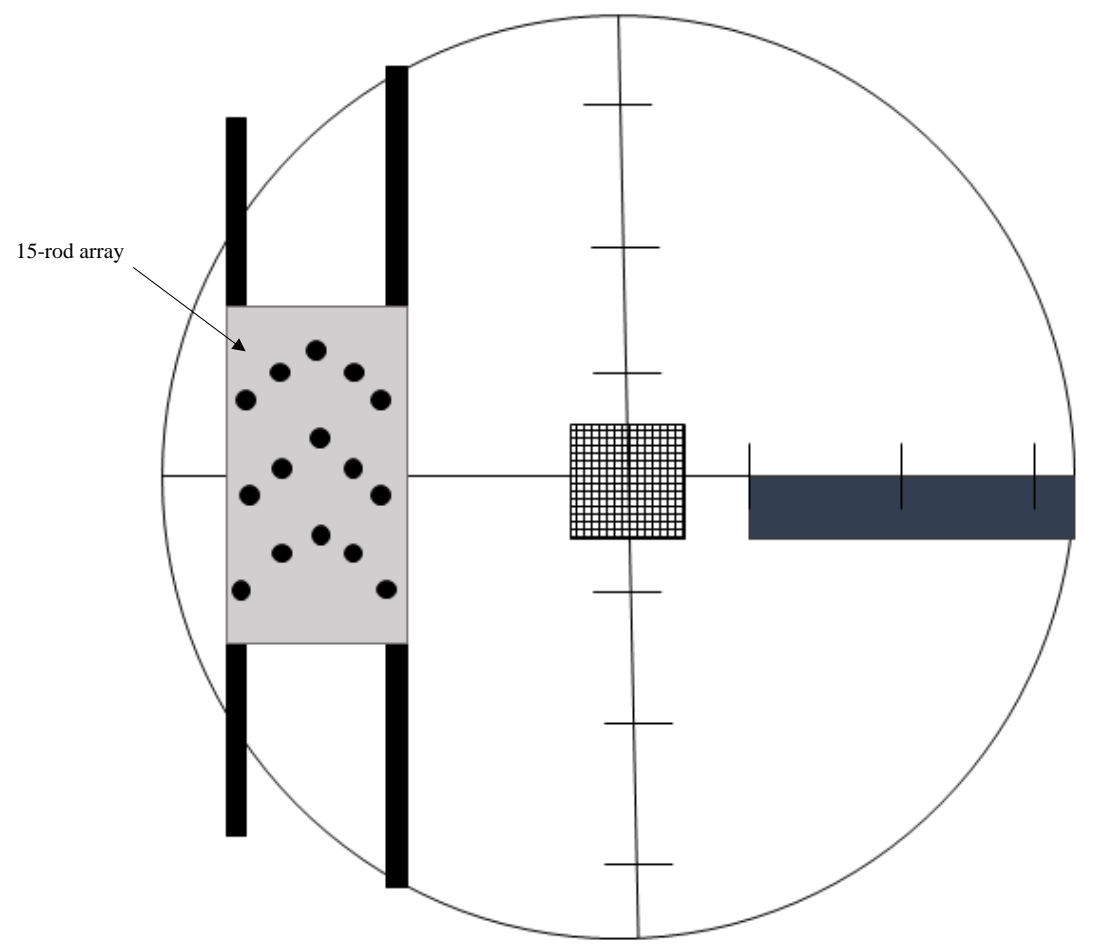

(b) 


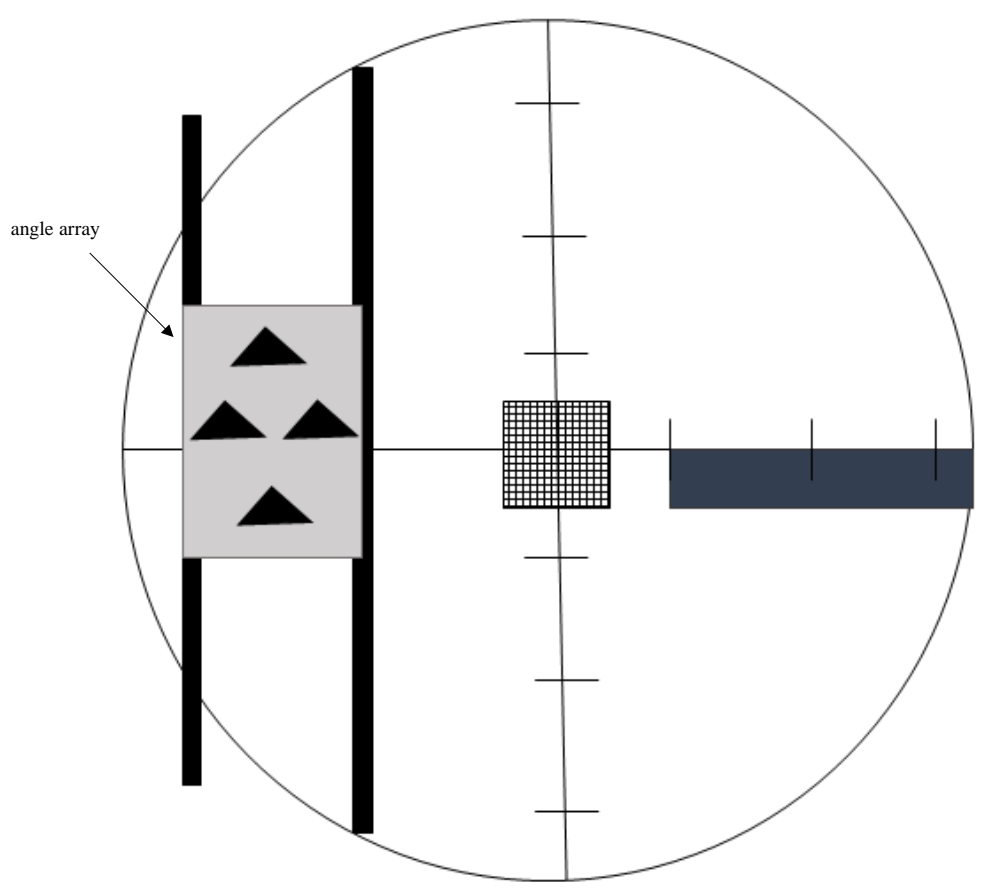

(c)

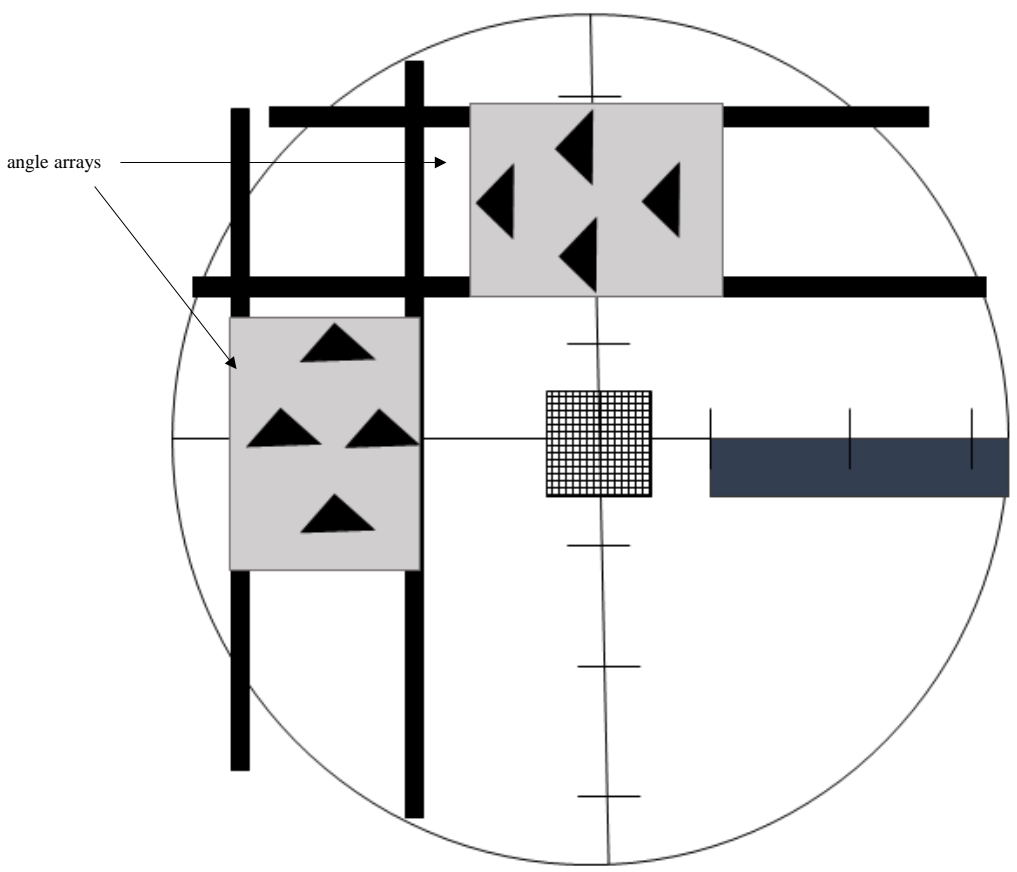

(d)

Figure 2. Experimental tanks with separate structure treatments, rod array (a), extended rod array (b), single angle array $(c)$, double angle array $(d)$.

length was labeled as an axis of $0^{\circ}, 90^{\circ}, 180^{\circ}$ or $270^{\circ}$. The spray bar was directly in front of the $0^{\circ}$ axis, and the structure was placed on the $180^{\circ}$ axis, on which no measurements were made. Each axis except for $180^{\circ}$ was split into three lengths of 30,60, and $90 \mathrm{~cm}$ from the central intersection of the strings. At each of these radii along the $0^{\circ}, 90^{\circ}$, and $270^{\circ}$ axes, three measurements were taken at 


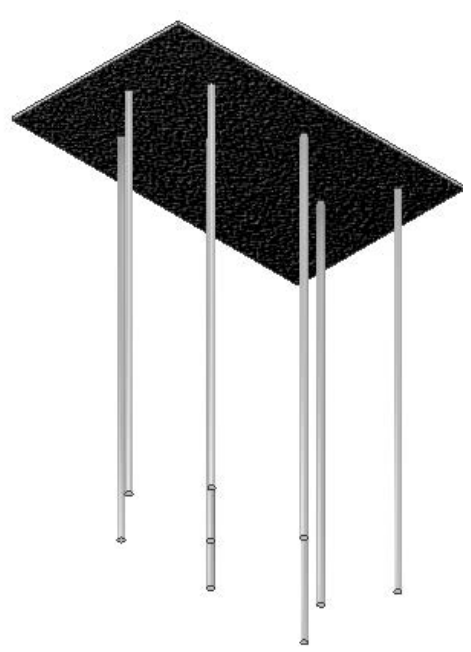

(a)

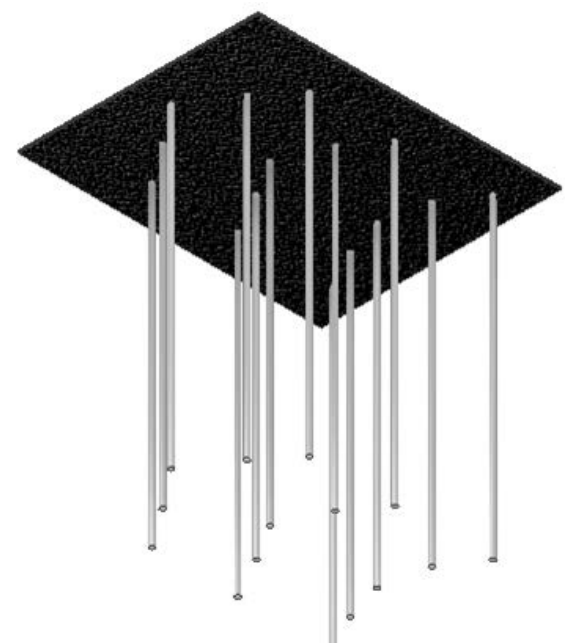

(b)

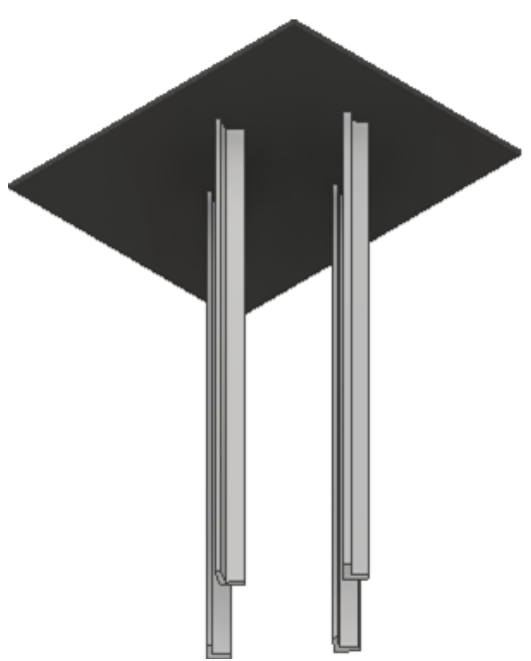

(c)

Figure 3. Diagrams of structures used in the tank with corrugated plastic tops. Structure (a) is the rod array $(27.30 \mathrm{~cm}$ by $46.96 \mathrm{~cm}),(\mathrm{b})$ is the extended rod array $(43.18 \mathrm{~cm}$ by $54.61 \mathrm{~cm})$, and $(\mathrm{c})$ is the angle array $(40.64 \mathrm{~cm}$ by $32.39 \mathrm{~cm})$. 


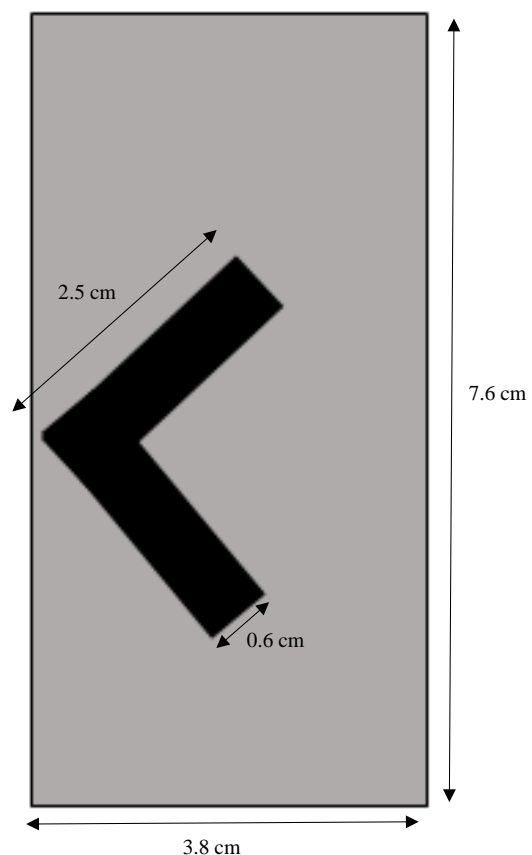

(a)

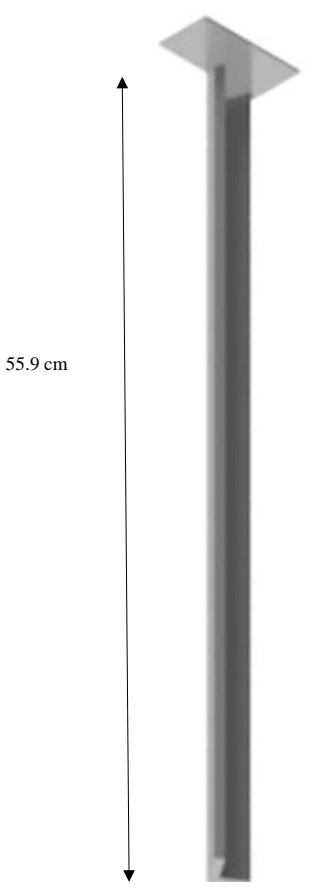

(b)

Figure 4. Close-up views of a single angle with dimensions. (a) bottom view of individual angle; (b) full view of angle structure

different depths from the surface: $10 \mathrm{~cm}, 30.5 \mathrm{~cm}$, and $51.8 \mathrm{~cm}$, as shown in Figure 5. Three replicate measurements were recorded at each location.

Data were initially analyzed by analysis of variance and covariance using the SPSS (9.0) statistical analysis program (SPSS, Chicago, Illinois, USA). Because of the large number of interactions, subsequent analysis used one-way analysis of 


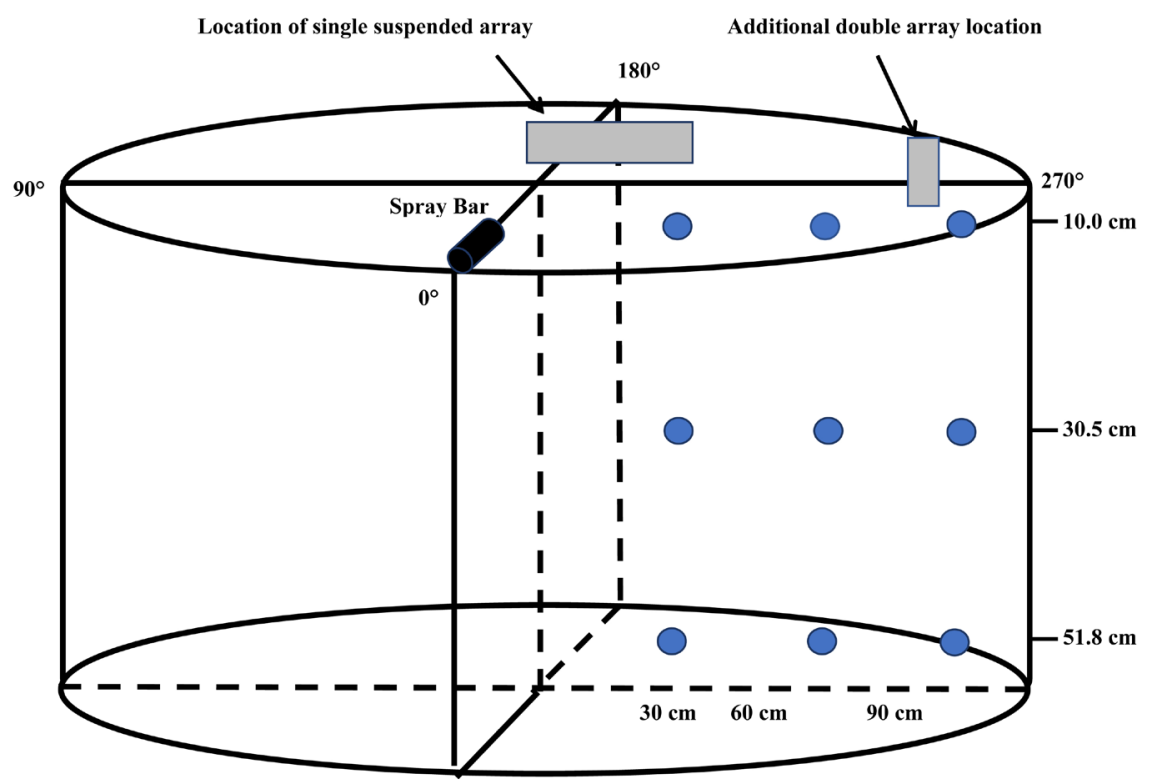

Figure 5. Diagram of a circular tank showing the location of the spray bar and sampling locations for one section (indicated by the blue circles).

variance and t-tests to examine just the effects of suspended arrays at each incoming water velocity. Tukey's mean comparison procedure was used for post-hoc analysis. The significance level for all tests was predetermined at $\mathrm{P}<0.05$.

\section{Results}

At both of the incoming water velocities $(18.3 \mathrm{~cm} / \mathrm{s}$ and $54.9 \mathrm{~cm} / \mathrm{s})$, overall water velocities throughout the tank were significantly reduced with the addition of any form of environmental enrichment in comparison to the control tanks devoid of structure (Table 2). In addition, the double angle enrichment treatment overall water velocities were significantly lower than the other three enrichment treatments. The lowest record velocity values of $1.0 \mathrm{~cm} / \mathrm{s}$ were observed in the extended rod and double angle enrichment treatments at an incoming velocity of $18.3 \mathrm{~cm} / \mathrm{s}$. The lowest recorded velocities at an incoming velocity of $54.9 \mathrm{~cm} / \mathrm{s}$ were $3.0 \mathrm{~cm} / \mathrm{s}$ in the extended rod and double angle treatments.

At both incoming velocities, water velocities at all depths were highest in the control treatment, with significant reductions in water velocities at all depths observed in the environmental enrichment treatments (Table 3). While there was considerable overlap, in general the lowest velocity readings at each depth were recorded in the double angle treatment. The pattern of significant reductions in velocity with the use of any environmental enrichment, with further significant reductions in the double angle treatment, was repeated when the data was combined for each radius (Table 4) and for each degree sampling location (Table 5).

Considerable variation in water velocity was observed at each specific sampling location in both the lower incoming velocity of $18.3 \mathrm{~cm} / \mathrm{s}$ (Table 6) and the higher incoming velocity of $54.9 \mathrm{~cm} / \mathrm{s}$ (Table 7). Despite this variation, 
Table 2. Mean (SE), minimum, and maximum water velocities $(\mathrm{cm} / \mathrm{s})$ from multiple sampling points within circular tanks containing different suspended environmental enrichment structures at two different incoming water velocities (means within each incoming velocity with different letters are significantly different, $\mathrm{P}<0.05$ ).

\begin{tabular}{cccccc}
\hline & & & \multicolumn{3}{c}{ Tank Velocity } \\
\hline Incoming Velocity & Structure & $\mathrm{N}$ & Overall & Minimum & Maximum \\
$18.3 \mathrm{~cm} / \mathrm{s}$ & None & 81 & $21.6(0.8) \mathrm{z}$ & 3.0 & 36.6 \\
& Rod & 81 & $15.1(0.6) \mathrm{x}$ & 3.0 & 24.4 \\
& Extended rod & 81 & $13.4(0.6) \mathrm{x}$ & 0.0 & 24.4 \\
& Single angle & 81 & $12.7(0.6) \mathrm{x}$ & 0.0 & 24.4 \\
& Double angle & 63 & $8.9(0.6) \mathrm{y}$ & 0.0 & 21.3 \\
$\mathrm{~F}_{(4,382)}=49.971 ; \mathrm{P}=0.001 \quad$ & & & & \\
$54.9 \mathrm{~cm} / \mathrm{s}$ & None & 81 & $44.5(1.2) \mathrm{z}$ & 18.3 & 67.1 \\
& Rod & 81 & $29.7(0.8) \mathrm{y}$ & 12.2 & 42.7 \\
& Extended rod & 81 & $25.4(0.8) \mathrm{x}$ & 3.0 & 39.6 \\
& Single angle & 81 & $25.4(0.9) \mathrm{x}$ & 6.1 & 36.6 \\
& Double angle & 63 & $17.5(0.6) \mathrm{w}$ & 3.0 & 30.5 \\
$\mathrm{~F}_{(4,382)}=120.343 ; \mathrm{P}=0.001$ & & & & \\
\hline
\end{tabular}

Table 3. Mean (SE) water velocities $(\mathrm{cm} / \mathrm{s})$ at three depths within circular tanks containing different suspended environmental enrichment structures at two different incoming water velocities $(\mathrm{N}=27$ except for Double Angle where $\mathrm{N}=24$; means within each incoming velocity in a row with different letters are significantly different, $\mathrm{P}<0.05)$.

\begin{tabular}{ccccccccc}
\hline \multicolumn{7}{c}{ Structure } \\
\hline Incoming Velocity & Depth & None & Rod & Extended Rod & Single Angle & Double Angle & $\mathrm{F}_{(4,124)}$ & $\mathrm{P}$ \\
\hline & Surface & $21.4(1.3) \mathrm{z}$ & $16.0(1.2) \mathrm{y}$ & $14.6(1.0) \mathrm{y}$ & $14.3(1.0) \mathrm{y}$ & $8.7(0.9) \mathrm{x}$ & 15.105 & 0.001 \\
\multirow{2}{*}{$18.9 \mathrm{~cm} / \mathrm{s}$} & Middle & $22.4(1.3) \mathrm{z}$ & $15.5(0.8) \mathrm{y}$ & $14.4(0.9) \mathrm{y}$ & $13.3(0.7) \mathrm{yx}$ & $10.0(1.1) \mathrm{x}$ & 20.677 & 0.001 \\
& Bottom & $21.0(1.4) \mathrm{z}$ & $13.7(1.1) \mathrm{y}$ & $11.1(1.1) \mathrm{yx}$ & $10.4(1.2) \mathrm{yx}$ & $7.8(1.1) \mathrm{x}$ & 17.267 & 0.001 \\
& Surface & $46.1(2.1) \mathrm{z}$ & $33.1(1.1) \mathrm{y}$ & $26.5(1.3) \mathrm{x}$ & $27.5(1.4) \mathrm{yx}$ & $19.0(1.0) \mathrm{w}$ & 44.280 & 0.001 \\
& Middle & $44.9(2.3) \mathrm{z}$ & $30.7(1.0) \mathrm{y}$ & $27.3(0.9) \mathrm{y}$ & $26.9(0.8) \mathrm{y}$ & $18.7(0.6) \mathrm{x}$ & 50.690 & 0.001 \\
& Bottom & $42.4(1.6) \mathrm{z}$ & $25.4(1.4) \mathrm{y}$ & $22.4(1.8) \mathrm{yx}$ & $21.8(1.8) \mathrm{yx}$ & $14.8(1.6) \mathrm{x}$ & 37.792 & 0.001 \\
\hline
\end{tabular}

Table 4. Mean (SE) water velocities $(\mathrm{cm} / \mathrm{s})$ at three radial locations within circular tanks containing different suspended environmental enrichment structures at two different incoming water velocities $(\mathrm{N}=27$ except for Double Angle at radius $=60$ and 90 where $\mathrm{N}=18$; means within each incoming velocity in a row with different letters are significantly different, $\mathrm{P}<0.05$ ).

\begin{tabular}{cccccccccc}
\hline \multicolumn{1}{c}{ Structure } \\
\hline Incoming Velocity & Radius & None & Rod & Extended Rod & Single Angle & Double Angle & $\mathrm{F}$ & $\mathrm{P}$ \\
\hline \multirow{2}{*}{$18.9 \mathrm{~cm} / \mathrm{s}$} & 30 & $15.5(1.2) \mathrm{z}$ & $10.8(0.7) \mathrm{y}$ & $9.1(0.9) \mathrm{yx}$ & $9.4(1.0) \mathrm{yx}$ & $6.2(0.9) \mathrm{x}$ & 12.997 & 0.001 \\
& 60 & $28.2(0.6) \mathrm{z}$ & $16.8(0.9) \mathrm{y}$ & $14.8(0.9) \mathrm{y}$ & $13.8(0.9) \mathrm{y}$ & $8.6(0.9) \mathrm{x}$ & 64.895 & 0.001 \\
& 90 & $21.1(0.7) \mathrm{z}$ & $17.5(0.9) \mathrm{y}$ & $16.1(0.9) \mathrm{yx}$ & $14.9(0.9) \mathrm{yx}$ & $13.0(0.7) \mathrm{x}$ & 11.582 & 0.001 \\
& 30 & $52.8(2.0) \mathrm{z}$ & $26.4(1.3) \mathrm{y}$ & $21.1(1.3) \mathrm{x}$ & $22.7(1.4) \mathrm{x}$ & $17.7(1.0) \mathrm{wx}$ & 96.558 & 0.001 \\
$54.9 \mathrm{~cm} / \mathrm{s}$ & 60 & $47.0(0.5) \mathrm{z}$ & $32.4(1.2) \mathrm{y}$ & $28.1(1.4) \mathrm{yx}$ & $25.9(1.6) \mathrm{x}$ & $16.6(1.7) \mathrm{w}$ & 67.898 & 0.001 \\
& 90 & $33.7(1.0) \mathrm{z}$ & $30.4(1.3) \mathrm{zy}$ & $27.0(1.2) \mathrm{y}$ & $27.7(1.3) \mathrm{y}$ & $18.1(1.2) \mathrm{x}$ & 19.223 & 0.001 \\
\hline
\end{tabular}


Table 5. Mean (SE) water velocities $(\mathrm{cm} / \mathrm{s})$ at three degree locations within circular tanks containing different suspended environmental enrichment structures at two different incoming water velocities ( $\mathrm{N}=27$ except for Double Angle at 270 where $\mathrm{N}=9$; means within each incoming velocity in a row with different letters are significantly different, $\mathrm{P}<0.05$ ).

\begin{tabular}{ccccccccc}
\hline \multicolumn{7}{c}{ Structure } \\
\hline Incoming Velocity & Degree & None & Rod & Extended Rod & Single Angle & Double Angle & $\mathrm{F}$ & $\mathrm{P}$ \\
\hline & $0^{\circ}$ & $21.7(1.0) \mathrm{z}$ & $15.8(1.0) \mathrm{y}$ & $14.2(0.6) \mathrm{y}$ & $13.5(1.0) \mathrm{yx}$ & $10.2(0.7) \mathrm{x}$ & 22.345 & 0.001 \\
$18.9 \mathrm{~cm} / \mathrm{s}$ & $90^{\circ}$ & $20.2(1.5) \mathrm{z}$ & $13.3(1.2) \mathrm{y}$ & $11.0(1.4) \mathrm{y}$ & $11.1(1.3) \mathrm{y}$ & $8.4(1.2) \mathrm{y}$ & 11.491 & 0.001 \\
& $270^{\circ}$ & $22.9(1.4) \mathrm{z}$ & $16.0(0.8) \mathrm{y}$ & $14.9(0.8) \mathrm{y}$ & $13.4(0.8) \mathrm{y}$ & $6.4(0.8) \mathrm{x}$ & 24.002 & 0.001 \\
& $0^{\circ}$ & $45.8(2.0) \mathrm{z}$ & $31.2(1.2) \mathrm{y}$ & $26.4(0.9) \mathrm{y}$ & $27.4(0.6) \mathrm{y}$ & $18.6(1.0) \mathrm{x}$ & 67.800 & 0.001 \\
$54.9 \mathrm{~cm} / \mathrm{s}$ & $90^{\circ}$ & $40.5(2.0) \mathrm{z}$ & $25.7(1.3) \mathrm{y}$ & $21.9(1.7) \mathrm{yx}$ & $21.4(1.8) \mathrm{yx}$ & $16.1(1.2) \mathrm{x}$ & 31.844 & 0.001 \\
& $270^{\circ}$ & $47.1(2.0) \mathrm{z}$ & $32.3(1.2) \mathrm{y}$ & $27.9(1.2) \mathrm{y}$ & $27.3(1.5) \mathrm{y}$ & $18.2(1.4) \mathrm{x}$ & 38.039 & 0.001 \\
\hline
\end{tabular}

Table 6. Mean (SE) water velocities $(\mathrm{cm} / \mathrm{s})$ at multiple locations within circular tanks containing different suspended environmental enrichment structures at an incoming water velocity of $18.3 \mathrm{~cm} / \mathrm{s}$ (means in a row with different letters are significantly different, $\mathrm{N}=3 ; \mathrm{P}<0.05)$.

\begin{tabular}{|c|c|c|c|c|c|c|c|c|c|}
\hline \multirow[b]{2}{*}{ Depth } & \multicolumn{4}{|c|}{ Measurement Location } & \multicolumn{3}{|c|}{ Structure } & \multirow[b]{2}{*}{$\mathrm{F}$} & \multirow[b]{2}{*}{$\mathrm{P}$} \\
\hline & Axis & Radii (cm) & None & Rod & Extended Rod & Single Angle & Double Angle & & \\
\hline \multirow{10}{*}{ Surface } & \multirow{3}{*}{$0^{\circ}$} & 30 & $16.3(2.0) \mathrm{z}$ & $15.2(1.8) \mathrm{z}$ & $14.2(1.0) \mathrm{z}$ & $15.2(0.0) \mathrm{z}$ & $14.2(1.0) \mathrm{z}$ & 0.389 & 0.812 \\
\hline & & 60 & $24.4(0.0) \mathrm{z}$ & $24.4(0.0) \mathrm{z}$ & $17.3(1.0) \mathrm{y}$ & $19.3(1.0) \mathrm{y}$ & $7.1(1.0) \mathrm{x}$ & 81.167 & 0.001 \\
\hline & & 90 & $18.3(0.0) \mathrm{z}$ & $18.3(0.0) \mathrm{z}$ & $15.2(0.0) \mathrm{y}$ & $18.3(0.0) \mathrm{z}$ & $11.2(1.0) \mathrm{x}$ & 47.500 & 0.001 \\
\hline & \multirow{3}{*}{$90^{\circ}$} & 30 & $10.2(2.0) \mathrm{z}$ & $8.1(2.7) \mathrm{z}$ & $7.1(1.0) \mathrm{z}$ & $7.1(2.7) \mathrm{z}$ & $3.0(1.8) \mathrm{z}$ & 1.477 & 0.280 \\
\hline & & 60 & $33.5(1.8) \mathrm{z}$ & $9.1(0.0) \mathrm{y}$ & $6.1(1.8) \mathrm{y}$ & $8.1(2.0) y$ & $6.1(1.8) \mathrm{y}$ & 51.654 & 0.001 \\
\hline & & 90 & $18.3(0.0) \mathrm{z}$ & $24.4(0.0) y$ & $23.4(1.0) \mathrm{y}$ & $21.3(1.8) \mathrm{zy}$ & $12.2(0.0) \mathrm{x}$ & 29.125 & 0.001 \\
\hline & \multirow{3}{*}{$270^{\circ}$} & 30 & $22.4(2.0) \mathrm{z}$ & $10.2(1.0) \mathrm{yx}$ & $12.2(0.0) \mathrm{yx}$ & $14.2(1.0) \mathrm{y}$ & $7.1(1.0) \mathrm{x}$ & 22.857 & 0.001 \\
\hline & & 60 & $28.4(1.0) \mathrm{z}$ & $18.3(0.0) y$ & $19.3(1.0) \mathrm{y}$ & $16.3(1.0) \mathrm{y}$ & - & 37.667 & 0.001 \\
\hline & & 90 & $21.3(0.0) \mathrm{z}$ & $16.3(1.0) \mathrm{z}$ & $16.3(1.0) \mathrm{z}$ & $9.1(1.8) \mathrm{y}$ & - & 19.467 & 0.001 \\
\hline & \multirow{3}{*}{$0^{\circ}$} & 30 & $21.3(1.8) \mathrm{z}$ & $10.2(2.0) \mathrm{y}$ & $13.2(2.0) \mathrm{y}$ & $9.1(3.0) \mathrm{y}$ & $10.2(1.0) \mathrm{y}$ & 5.786 & 0.011 \\
\hline \multirow{8}{*}{ Middle } & & 60 & $28.4(1.0) \mathrm{z}$ & $17.3(1.0) y$ & $15.2(1.8) \mathrm{y}$ & $15.2(1.8) y$ & $9.1(0.0) \mathrm{x}$ & 30.125 & 0.001 \\
\hline & & 90 & $24.4(0.0) \mathrm{z}$ & $20.3(1.0)$ zy & $17.3(1.0) \mathrm{yx}$ & $17.3(1.0) y x$ & $14.2(1.0) \mathrm{x}$ & 17.875 & 0.001 \\
\hline & \multirow{3}{*}{$90^{\circ}$} & 30 & $10.2(1.0) \mathrm{z}$ & $10.2(1.0) \mathrm{z}$ & $5.1(1.0) y x$ & $7.1(1.0) \mathrm{z}$ & $1.0(1.0) \mathrm{x}$ & 14.300 & 0.001 \\
\hline & & 60 & $30.5(0.0) \mathrm{z}$ & $18.3(0.0) \mathrm{y}$ & $18.3(1.8) \mathrm{y}$ & $15.2(0.0) \mathrm{y}$ & $9.1(0.0) \mathrm{x}$ & 97.505 & 0.001 \\
\hline & & 90 & $15.2(0.0) \mathrm{zx}$ & $11.2(1.0) \mathrm{y}$ & $14.2(1.0) \mathrm{yx}$ & $11.2(1.0) \mathrm{y}$ & $18.3(0.0) \mathrm{z}$ & 14.500 & 0.001 \\
\hline & \multirow{3}{*}{$270^{\circ}$} & 30 & $20.3(2.0) \mathrm{z}$ & $16.3(1.0)$ zy & $11.2(2.0) \mathrm{yx}$ & $14.2(1.0) \mathrm{zyx}$ & $8.1(1.0) \mathrm{x}$ & 9.636 & 0.002 \\
\hline & & 60 & $29.5(1.0) \mathrm{z}$ & $16.3(1.0) \mathrm{y}$ & $18.3(0.0) \mathrm{y}$ & $15.2(0.0) \mathrm{y}$ & - & 83.333 & 0.001 \\
\hline & & 90 & $21.3(1.8) \mathrm{z}$ & $19.3(1.0) \mathrm{z}$ & $17.3(1.0) \mathrm{z}$ & $15.2(0.0) \mathrm{z}$ & - & 5.333 & 0.026 \\
\hline \multirow{9}{*}{ Bottom } & \multirow{3}{*}{$0^{\circ}$} & 30 & $12.2(1.8) \mathrm{z}$ & $10.2(1.0) \mathrm{z}$ & $10.2(1.0) \mathrm{z}$ & $6.1(3.5) \mathrm{z}$ & $7.1(2.7) \mathrm{z}$ & 1.250 & 0.351 \\
\hline & & 60 & $24.4(0.0) \mathrm{z}$ & $9.1(0.0) \mathrm{y}$ & $9.1(0.0) y$ & $7.1(1.0) \mathrm{yx}$ & $5.1(1.0) \mathrm{x}$ & 143.000 & 0.001 \\
\hline & & 90 & $25.4(1.0) \mathrm{z}$ & $17.3(1.0) y$ & $16.3(1.0) \mathrm{y}$ & $14.2(1.0) \mathrm{y}$ & $13.2(1.0) \mathrm{y}$ & 22.500 & 0.001 \\
\hline & \multirow{3}{*}{$90^{\circ}$} & 30 & $19.3(1.0) \mathrm{z}$ & $8.1(1.0) \mathrm{y}$ & $1.0(1.0) \mathrm{x}$ & $2.0(2.0) \mathrm{x}$ & $1.0(1.0) \mathrm{x}$ & 37.313 & 0.001 \\
\hline & & 60 & $24.4(0.0) \mathrm{z}$ & $21.3(0.0) y$ & $17.3(1.0) \mathrm{xw}$ & $19.3(1.0) \mathrm{yx}$ & $15.2(0.0) \mathrm{w}$ & 30.500 & 0.001 \\
\hline & & 90 & $20.3(1.0) \mathrm{z}$ & $9.1(1.8) y$ & $6.1(1.8) y$ & $8.1(2.0) \mathrm{y}$ & $9.1(0.0) \mathrm{y}$ & 13.773 & 0.001 \\
\hline & \multirow{3}{*}{$270^{\circ}$} & 30 & $7.1(2.7) \mathrm{z}$ & $9.1(1.8) \mathrm{z}$ & $8.1(1.0) \mathrm{z}$ & $9.1(1.8) \mathrm{z}$ & $4.1(1.0) \mathrm{z}$ & 1.433 & 0.293 \\
\hline & & 60 & $30.5(0.0) \mathrm{z}$ & $17.3(1.0) y$ & $12.2(1.8) \mathrm{yx}$ & $8.1(1.0) \mathrm{x}$ & - & 73.267 & 0.001 \\
\hline & & 90 & $25.4(1.0) \mathrm{z}$ & $21.3(1.8)$ zy & $19.3(1.0) \mathrm{y}$ & $19.3(1.0) \mathrm{y}$ & - & 5.333 & 0.026 \\
\hline
\end{tabular}


Table 7. Mean (SE) water velocities $(\mathrm{cm} / \mathrm{s})$ at multiple locations within circular tanks containing different suspended environmental enrichment structures at an incoming water velocity of $54.9 \mathrm{~cm} / \mathrm{s}$ (means in a row with different letters are significantly different, $\mathrm{N}=3 ; \mathrm{P}<0.05)$.

\begin{tabular}{|c|c|c|c|c|c|c|c|c|c|}
\hline \multirow[b]{2}{*}{ Depth } & \multicolumn{4}{|c|}{ Measurement Location } & \multicolumn{3}{|c|}{ Structure } & \multirow[b]{2}{*}{$\mathrm{F}$} & \multirow[b]{2}{*}{$\mathrm{P}$} \\
\hline & Axis & Radii (cm) & None & Rod & Extended Rod & Single Angle & Double Angle & & \\
\hline \multirow{9}{*}{ Surface } & \multirow{3}{*}{$0^{\circ}$} & 30 & $58.9(1.0) \mathrm{z}$ & $30.5(3.5) y$ & $21.3(3.0) y$ & $26.4(1.0) y$ & $21.3(0.0) y$ & 51.891 & 0.001 \\
\hline & & 60 & $48.8(0.0) \mathrm{z}$ & $39.6(1.8) y$ & $30.5(3.5) \mathrm{x}$ & $30.5(0.0) \mathrm{x}$ & $19.3(1.0) \mathrm{w}$ & 37.094 & 0.001 \\
\hline & & 90 & $33.5(0.0) \mathrm{z}$ & $34.5(1.0) \mathrm{z}$ & $28.4(1.0) y$ & $28.4(1.0) y$ & $26.4(1.0) \mathrm{y}$ & 15.250 & 0.001 \\
\hline & \multirow{3}{*}{$90^{\circ}$} & 30 & $50.8(1.0) \mathrm{z}$ & $22.4(1.0) y$ & $19.3(1.0) y$ & $19.3(2.0) y$ & $17.3(1.0) y$ & 120.188 & 0.001 \\
\hline & & 60 & $9.0(2.0) \mathrm{z}$ & $33.5(0.0) y$ & $18.3(1.8) \mathrm{x}$ & $13.2(1.0) \mathrm{xw}$ & $9.1(0.0) \mathrm{w}$ & 155.000 & 0.001 \\
\hline & & 90 & $27.4(1.8) \mathrm{z}$ & $29.5(1.0) y$ & $29.5(1.0) y$ & $35.6(1.0) \mathrm{z}$ & $18.3(0.0) \mathrm{x}$ & 31.500 & 0.001 \\
\hline & \multirow{3}{*}{$270^{\circ}$} & 30 & $61.0(0.0) \mathrm{z}$ & $37.6(2.0) y$ & $27.4(1.8) \mathrm{xw}$ & $32.5(2.0) y x$ & $21.3(1.8) \mathrm{w}$ & 80.107 & 0.000 \\
\hline & & 60 & $48.8(0.0) \mathrm{z}$ & $39.6(1.8) y$ & $38.6(1.0) y$ & $35.6(1.8) y$ & - & 25.067 & 0.001 \\
\hline & & 90 & $37.6(1.0) \mathrm{z}$ & $30.5(0.0) y$ & $25.4(1.0) \mathrm{x}$ & $26.4(1.0) x$ & - & 39.556 & 0.000 \\
\hline \multirow{9}{*}{ Middle } & \multirow{3}{*}{$0^{\circ}$} & 30 & $63.0(1.0) \mathrm{z}$ & $25.4(1.0) \mathrm{yx}$ & $25.4(2.0) \mathrm{yx}$ & $30.5(0.0) y$ & $21.3(0.0) \mathrm{x}$ & 233.583 & 0.001 \\
\hline & & 60 & $46.7(1.0) \mathrm{z}$ & $34.5(4.1) y$ & $29.5(1.0) y$ & $27.4(1.8) y$ & $16.3(1.0) \mathrm{x}$ & 27.114 & 0.001 \\
\hline & & 90 & $35.6(1.0) \mathrm{z}$ & 33.5 (1.8) zy & $31.5(1.0) \mathrm{zy}$ & $28.4(1.0) y$ & $20.3(1.0) x$ & 24.500 & 0.001 \\
\hline & \multirow{3}{*}{$90^{\circ}$} & 30 & $46.7(4.4) \mathrm{z}$ & $25.4(1.0) y$ & $23.4(2.7) \mathrm{y}$ & $23.4(1.0) y$ & $18.3(1.8) y$ & 19.724 & 0.001 \\
\hline & & 60 & $47.8(1.0) \mathrm{z}$ & $31.5(2.7) y$ & $30.5(0.0) y$ & $26.4(1.0) y$ & $18.3(1.8) \mathrm{x}$ & 46.792 & 0.001 \\
\hline & & 90 & $23.4(2.7) \mathrm{z}$ & $23.4(2.7) \mathrm{z}$ & $18.3(1.8) \mathrm{z}$ & $20.3(2.7) \mathrm{z}$ & $17.3(1.0) \mathrm{z}$ & 1.594 & 0.264 \\
\hline & \multirow{3}{*}{$270^{\circ}$} & 30 & $58.9(2.0) \mathrm{z}$ & $32.5(1.0) y$ & $27.4(0.0) y x$ & $24.4(0.0) \mathrm{xw}$ & $19.3(2.7) \mathrm{w}$ & 97.292 & 0.001 \\
\hline & & 60 & $45.7(0.0) \mathrm{z}$ & $33.5(1.8) y$ & $29.5(1.0) \mathrm{y}$ & $29.5(2.0) \mathrm{y}$ & - & 28.677 & 0.001 \\
\hline & & 90 & $36.6(0.0) \mathrm{z}$ & $36.6(0.0) \mathrm{z}$ & $30.5(0.0) y$ & $31.5(1.0) y$ & - & 41.000 & 0.001 \\
\hline \multirow{9}{*}{ Bottom } & \multirow{3}{*}{$0^{\circ}$} & 30 & $42.7(5.3) \mathrm{z}$ & $25.4(3.7) y$ & $20.3(1.0) y$ & $22.4(2.0) y$ & $19.3(1.0) y$ & 9.707 & 0.002 \\
\hline & & 60 & $44.7(1.0) \mathrm{z}$ & $23.4(2.0) \mathrm{y}$ & $22.4(1.0) \mathrm{y}$ & $24.4(0.0) y$ & $8.1(1.8) \mathrm{x}$ & 118.000 & 0.001 \\
\hline & & 90 & $38.6(1.0) \mathrm{z}$ & $33.5(3.0) \mathrm{zy}$ & $28.4(1.0) y$ & $28.4(1.0) y$ & $15.2(0.0) \mathrm{x}$ & 30.542 & 0.001 \\
\hline & \multirow{3}{*}{$90^{\circ}$} & 30 & $40.6(7.3) \mathrm{z}$ & $17.3(3.7) y$ & $6.1(1.8) y$ & $7.1(1.0) y$ & $7.1(2.7) y$ & 13.770 & 0.001 \\
\hline & & 60 & $45.7(3.5) \mathrm{z}$ & $31.5(1.0) y$ & $35.6(1.0) y$ & $33.5(1.8) y$ & $28.4(1.0) \mathrm{y}$ & 11.611 & 0.001 \\
\hline & & 90 & $34.5(1.0) \mathrm{z}$ & $17.3(2.0) y$ & $16.3(1.0) y$ & $14.2(1.0) \mathrm{y}$ & $11.2(2.0) \mathrm{y}$ & 36.955 & 0.001 \\
\hline & \multirow{3}{*}{$270^{\circ}$} & 30 & $52.8(8.9) \mathrm{z}$ & $21.3(3.0) y$ & $19.3(1.0) y$ & $18.3(0.0) \mathrm{y}$ & $14.2(1.0) \mathrm{y}$ & 13.661 & 0.001 \\
\hline & & 60 & $46.7(2.0) \mathrm{z}$ & $24.4(1.8) y$ & $18.3(1.8) y$ & $12.2(1.8) \mathrm{x}$ & - & 67.692 & 0.001 \\
\hline & & 90 & $35.6(1.0) \mathrm{z}$ & $34.5(1.0) \mathrm{z}$ & $34.5(1.0) \mathrm{z}$ & $35.6(1.0) \mathrm{z}$ & - & 0.333 & 0.802 \\
\hline
\end{tabular}

significantly lower velocities were observed at nearly every sampling location with the addition of any environmental enrichment to the circular tank, as indicated in the cross-sectional velocity profiles shown in Figures 6-11. The double angle array consistently produced the lowest velocities among the environmental enrichment treatments at each incoming velocity, except at $30 \mathrm{~cm}$ radii on the surface and middle depths. In addition, the velocities among the enrichment 


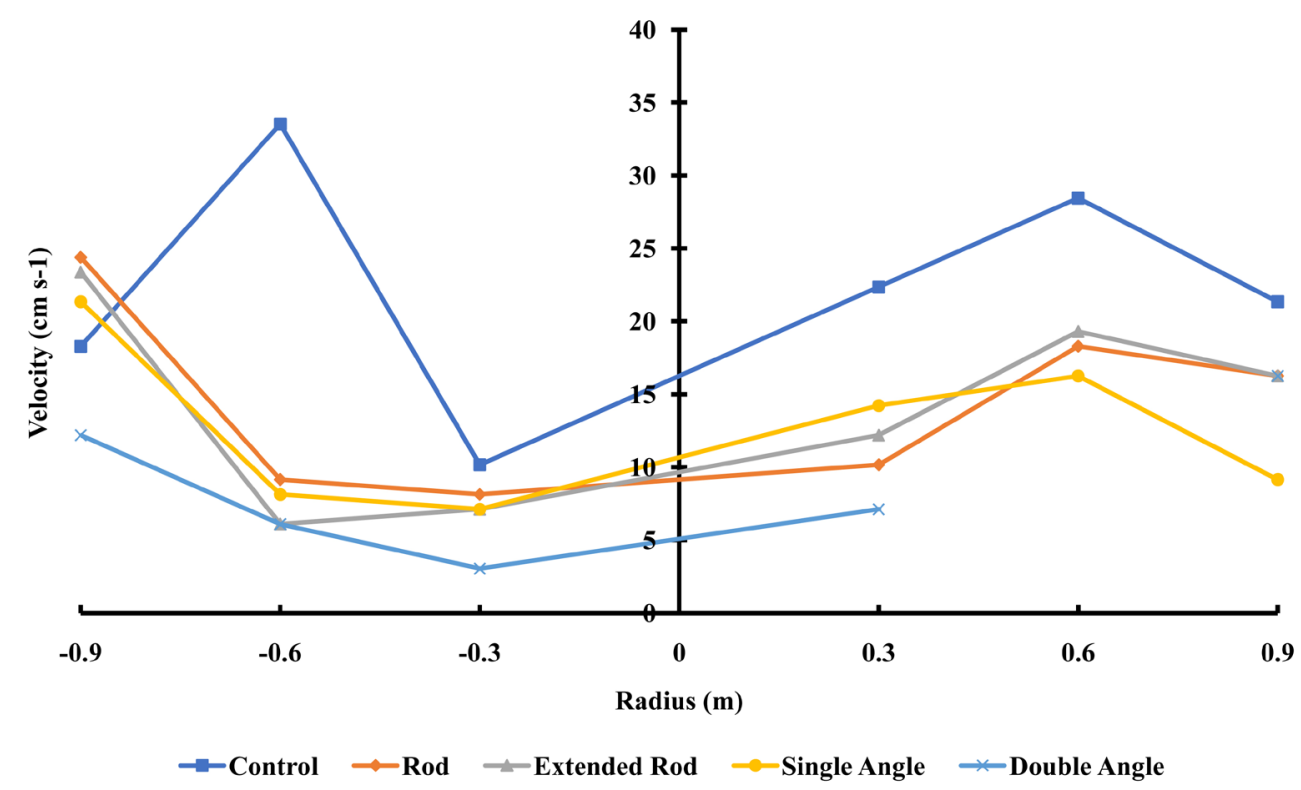

Figure 6. Cross section velocity profiles at a depth of $10 \mathrm{~cm}$ (surface) of a $1.8 \mathrm{~m}$ diameter circular tank containing different types of suspended environmental enrichment arrays at an incoming velocity of $18.3 \mathrm{~cm} / \mathrm{s}$. The cross section was perpendicular to the location of the inlet water spraybar and suspended enrichment. Part of the double angle enrichment was located at 0.6 and $0.9 \mathrm{~m}$, precluding velocity measurement.

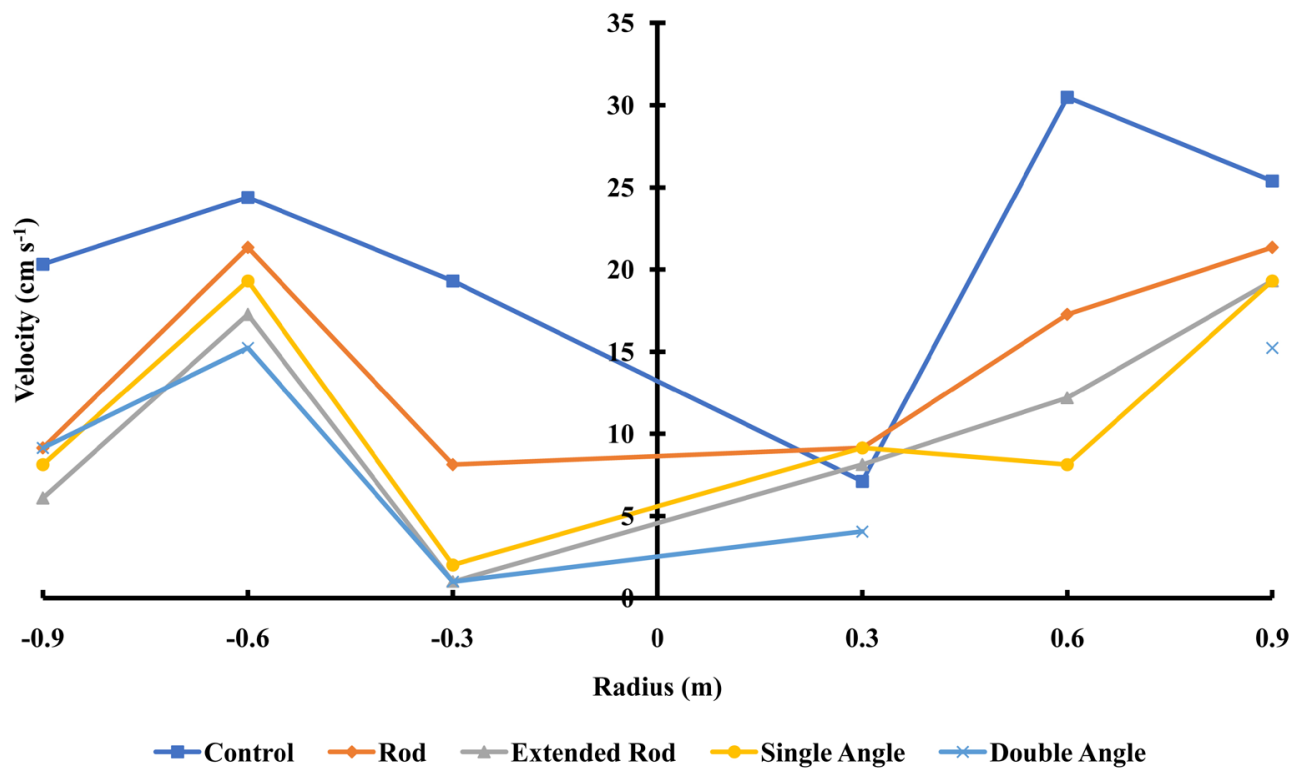

Figure 7. Cross section velocity profiles at a depth of $30.5 \mathrm{~cm}$ (mid-depth) of a $1.8 \mathrm{~m}$ diameter circular tank containing different types of suspended environmental enrichment arrays at an incoming velocity of $18.3 \mathrm{~cm} / \mathrm{s}$. The cross section was perpendicular to the location of the inlet water spraybar and suspended enrichment. Part of the double angle enrichment was located at 0.6 and $0.9 \mathrm{~m}$, precluding velocity measurement.

treatments were similar at both the surface and bottom at $0^{\circ}$, radii $30 \mathrm{~cm}$ at the incoming velocity of $18.3 \mathrm{~cm} / \mathrm{s}$, and radii $90 \mathrm{~cm}$ at $90^{\circ}$ at the surface and $270^{\circ}$ at the bottom of the $54.9 \mathrm{~cm} / \mathrm{s}$ incoming velocity. 


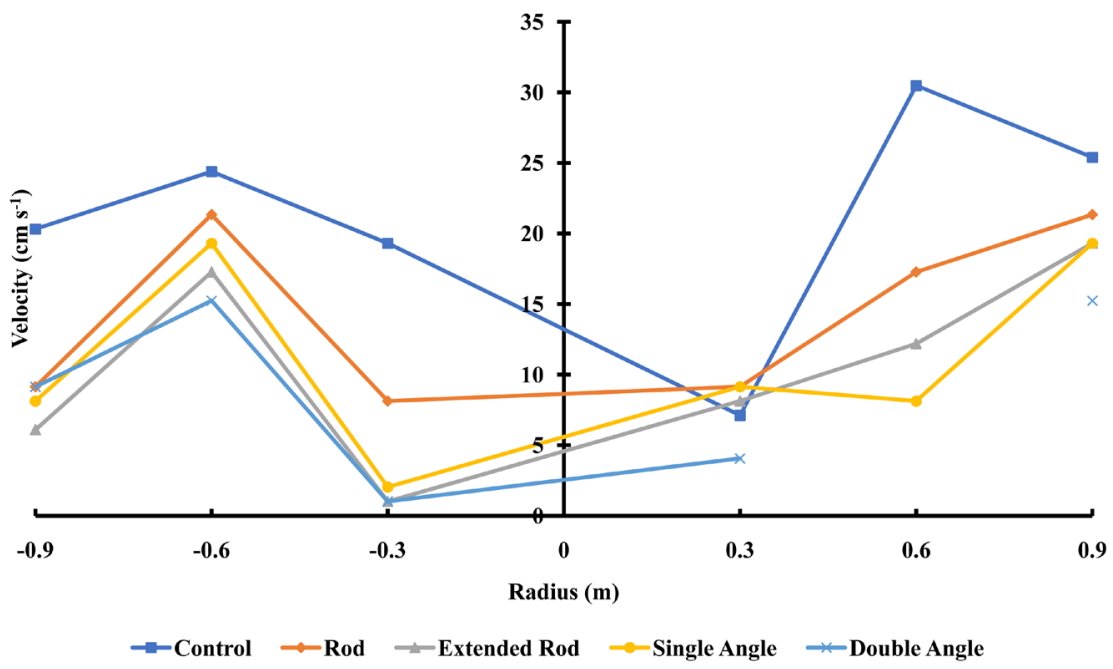

Figure 8. Cross section velocity profiles at a depth of $51.8 \mathrm{~cm}$ (bottom) of a $1.8 \mathrm{~m}$ diameter circular tank containing different types of suspended environmental enrichment arrays at an incoming velocity of $18.3 \mathrm{~cm} / \mathrm{s}$. The cross section was perpendicular to the location of the inlet water spraybar and suspended enrichment. Part of the double angle enrichment was located at 0.6 and $0.9 \mathrm{~m}$, precluding velocity measurement.

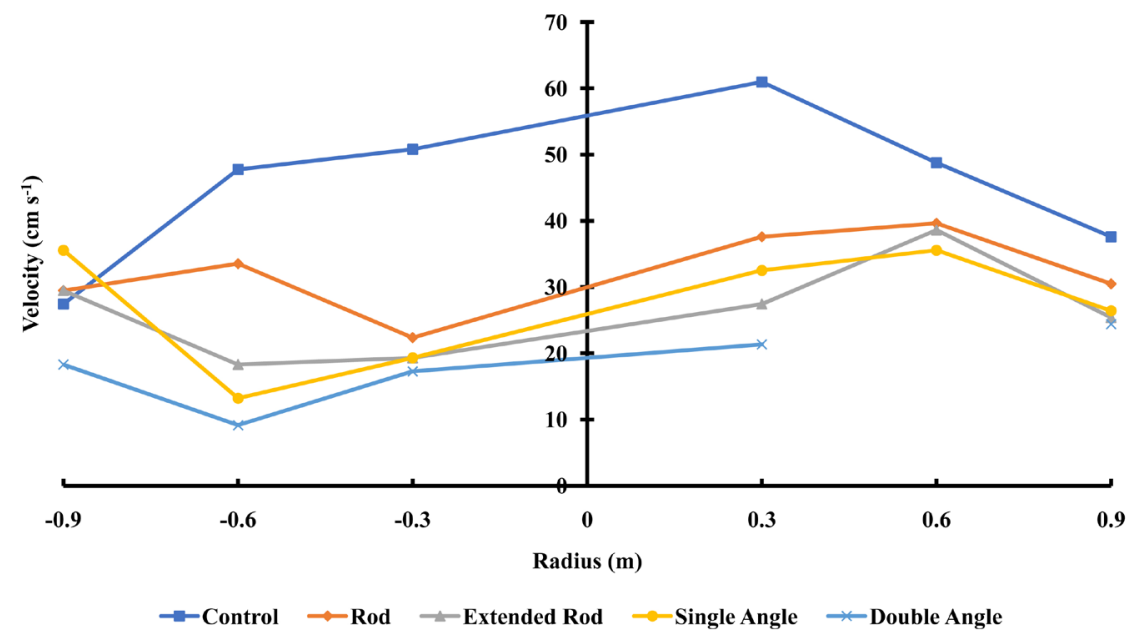

Figure 9. Cross section velocity profiles at a depth of $10 \mathrm{~cm}$ (surface) of a $1.8 \mathrm{~m}$ diameter circular tank containing different types of suspended environmental enrichment arrays at an incoming velocity of $54.9 \mathrm{~cm} / \mathrm{s}$. The cross section was perpendicular to the location of the inlet water spraybar and suspended enrichment. Part of the double angle enrichment was located at 0.6 and $0.9 \mathrm{~m}$, precluding velocity measurement.

\section{Discussion}

The significant in-tank velocity reductions resulting from the addition of vertically-suspended structures observed in this study support the results previously reported [10]. The decrease in velocity at nearly every sampling location occurred despite the minimal space occupied by the structures in the tank. The most dramatic impacts on velocity were observed with the double angle treatment, yet these two arrays, containing a total of only eight suspended angles, occupied less than $0.009 \%$ of the total tank volume. 


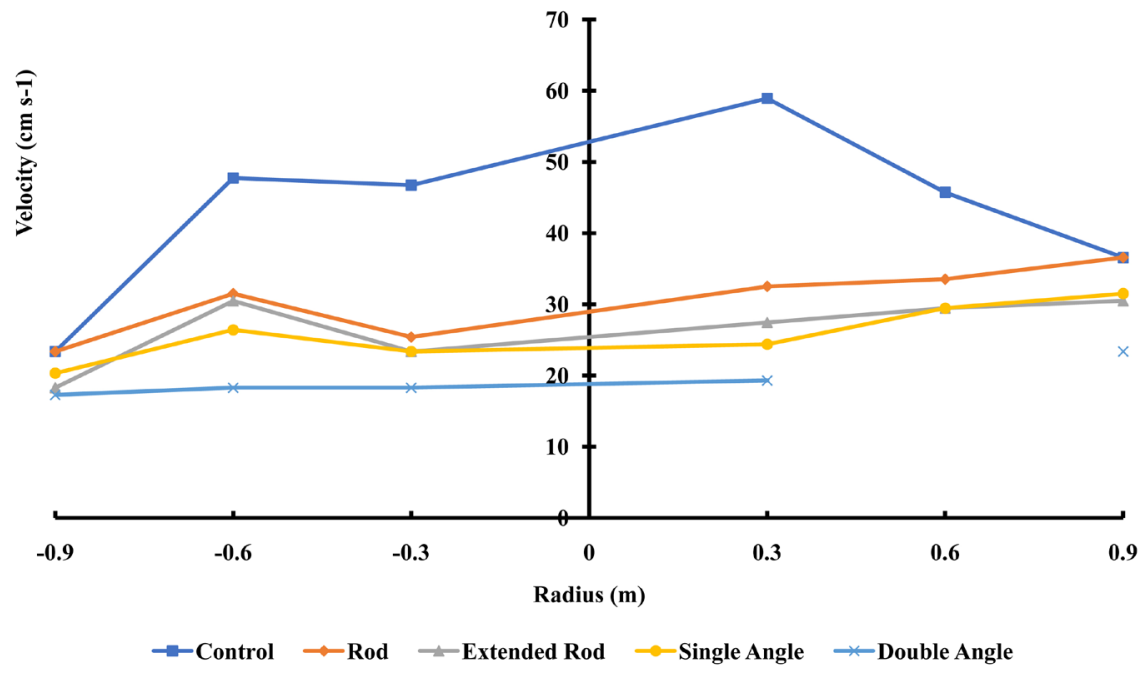

Figure 10. Cross section velocity profiles at a depth of $30.5 \mathrm{~cm}$ (mid-depth) of a $1.8 \mathrm{~m}$ diameter circular tank containing different types of suspended environmental enrichment arrays at an incoming velocity of $54.9 \mathrm{~cm} / \mathrm{s}$. The cross section was perpendicular to the location of the inlet water spraybar and suspended enrichment. Part of the double angle enrichment was located at 0.6 and $0.9 \mathrm{~m}$, precluding velocity measurement.

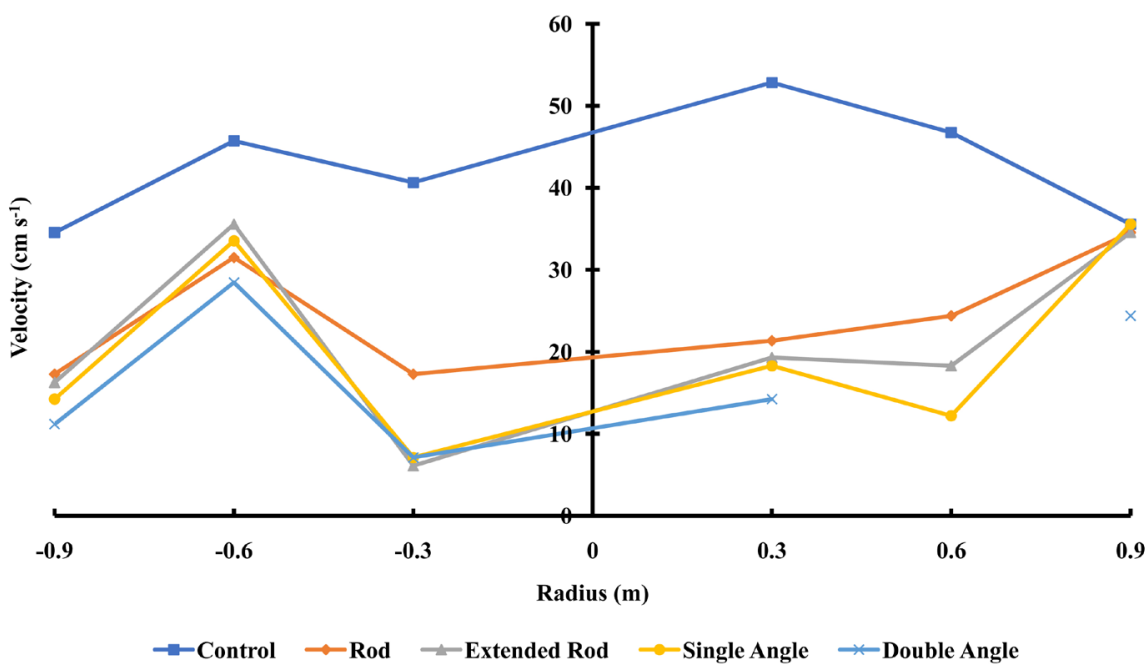

Figure 11. Cross section velocity profiles at a depth of $51.8 \mathrm{~cm}$ (bottom) of a $1.8 \mathrm{~m} \mathrm{di-}$ ameter circular tank containing different types of suspended environmental enrichment arrays at an incoming velocity of $54.9 \mathrm{~cm} / \mathrm{s}$. The cross section was perpendicular to the location of the inlet water spraybar and suspended enrichment. Part of the double angle enrichment was located at 0.6 and $0.9 \mathrm{~m}$, precluding velocity measurement.

At the incoming velocity of $18.9 \mathrm{~cm} / \mathrm{s}$, the extended rod, single angle, and double angle treatments all produced in-tank velocities below the limit of 12 to $15 \mathrm{~cm} / \mathrm{s}$ where tank self-cleaning may start to become affected [1]. Indeed, the bottom velocity of $7.8 \mathrm{~cm} / \mathrm{s}$ created by the double angle environmental enrichment array was actually below the $8 \mathrm{~cm} / \mathrm{s}$ threshold were hydraulic self-cleaning completely ceases [1]. At the higher incoming velocity of $54.9 \mathrm{~cm} / \mathrm{s}$, the in-tank velocities observed in all of the treatments indicated that the self-cleaning nature 
of the circular tanks was not affected by the addition of vertically-suspended environmental enrichment.

Circular tank velocities increase from the center of the tank outward, with the fastest in-tank velocities occurring nearest to the outerwall [4]. Circular tank velocities are highest near the outside of the tank, as well as being higher at the top of the tank [1]. Similar velocity patterns were observed in this study, except with the double angle treatment at the lower incoming water velocity. In this specific treatment, the mean velocity of $8.7 \mathrm{~cm} / \mathrm{s}$ at the surface was less than the $10.0 \mathrm{~cm} / \mathrm{s}$ velocity observed at mid-depth.

A large central vortex in the middle of circular tanks has been previously been described [35]. An irrotational zone in the center of circular tanks has also noted [2]. This was observed in this study in all of the treatments at both incoming velocities, despite the in-tank velocity reductions resulting from the inclusion of vertically-suspended environmental enrichment structures.

The increase in fish growth and rearing efficiencies using arrays of suspended objects in circular tanks is well documented [27] [28] [29] [30] [31]. These fish rearing benefits are likely at least partially because of the dramatic in-tank velocity changes due to the suspended environmental enrichment. The lower velocity areas created by the arrays may function as favorable bioenergetic microhabitats, allowing fish to avoid the more energy-consuming, high-velocity areas during non-feeding periods [36]. Fish likely benefit from these rest intervals. Indeed, although higher velocity-induced exercise is generally considered beneficial during fish rearing [5] [6] [32] [33], long-term exposure to higher velocities (continual exercise) has recently been shown to be detrimental to fish growth [7] [8]. Thus, the suspended arrays may be allowing the fish to minimize their energy expenditures during feeding, and also benefit from periodic exercise in the higher-velocity areas within the tank [36] [37].

In addition to the potential bioenergetic benefits, the suspended arrays may also be positively influencing in-tank water chemistry parameters, such as the distribution and concentration of dissolved oxygen. Although velocities and water quality are more uniformly distributed in circular tanks compared to rectangular rearing units [3] [4], there is obviously still within-tank variation [9] [13] [14]. The alterations in circular tank velocity patterns from suspended enrichment observed in this study and previously described [9] may make dissolved oxygen levels more favorable throughout the entire tanks, thereby leading to improved fish growth and feeding efficiency [38] [39].

Circular tank velocity profiles can be influenced by multiple factors, including tank size, water inlets and outlets, incoming water velocities, and the number and size of fish in the tank [4] [9] [12] [13] [14]. Even changing from a horizontal incoming-water spray bar, like that used in this study, to a vertical spray bar would impact the velocity profile [1]. Thus, the results of this study may be unique to the $1.8-\mathrm{m}$ diameter circular tanks, incoming water velocities, and vertically-suspended environmental enrichment arrays used in this study. Addi- 
tional research could focus on any of these variables. In addition, velocity profiles with vertically-suspended environmental enrichment while fish are present would be extremely beneficial. However, as yet, no studies have been undertaken to ascertain the impact of vertically-suspended structure on fish behavior; structure-induced changes in foraging, resting, or swimming behavior would likely dramatically influence in-tank velocities

\section{Conflicts of Interest}

The authors declare no conflicts of interest regarding the publication of this paper.

\section{References}

[1] Lekang, O.-I. (2013) Aquaculture Engineering. 2nd Edition, Wiley-Blackwell, Chichester. https://doi.org/10.1002/9781118496077

[2] Timmons, M.B., Summerfelt, S.T. and Vinci, B.J. (1998) Review of Circular Tank Technology and Management. Aquacultural Engineering, 18, 51-69. https://doi.org/10.1016/S0144-8609(98)00023-5

[3] Duarte S., Reig, L., Masaló, I., Blanco, M. and Oca, J. (2011) Influence of Tank Geometry and Flow Pattern in Fish Distribution. Aquacultural Engineering, 44, 48-54. https://doi.org/10.1016/j.aquaeng.2010.12.002

[4] Oca, J. and Masalo, I. (2013) Flow Pattern in Aquaculture Circular Tanks: Influence of Flow Rate, Water Depth, and Water Inlet \& Outlet Features. Aquacultural Engineering, 52, 65-72. https://doi.org/10.1016/j.aquaeng.2012.09.002

[5] Parker, T.M. and Barnes, M.E. (2014) Rearing Velocity Impacts on Landlocked Fall Chinook Salmon (Oncorhynchus tshawytscha) Growth, Condition, and Survival. Open Journal of Animal Sciences, 4, 244-252. https://doi.org/10.4236/ojas.2014.45031

[6] Parker, T.M. and Barnes, M.E. (2015) Effects of Different Water Velocities on the Hatchery Rearing Performance and Recovery from Transportation of Rainbow Trout Fed Two Different Rations. Transactions of the American Fisheries Society, 144, 882-890. https://doi.org/10.1080/00028487.2015.1047533

[7] Voorhees, J.M., Barnes, M.E., Chipps, S.R. and Brown, M.L. (2018) Rearing Performance of Juvenile Brown Trout (Salmo trutta) Subjected to Exercise and Dietary Bioprocessed Soybean Meal. Open Journal of Animal Sciences, 8, 303-328. https://doi.org/10.4236/ojas.2018.83023

[8] Voorhees, J.M., Barnes, M.E., Chipps, S.R. and Brown, M.L. (2018) Dietary Bioprocessed Soybean Meal Does Not Affect the Growth of Exercised Rainbow Trout (Oncorhynchus mykiss). Journal of Animal Research and Nutrition, 3, 6.

[9] Gorle, J.M.R., Terjesen B.F. and Summerfelt, S.F. (2018) Hydrodynamics of Octagonal Culture Tanks with Cornell-Type Dual-Drain System. Computers and Electronic in Agriculture, 151, 354-364. https://doi.org/10.1016/j.compag.2018.06.012

[10] Moine, J., Barnes M.E., Kientz J. and Simpson G. (2016) Flow Patterns in Circular Rearing Tanks Containing Vertical Structure. Journal of Fisheries and Livestock Production, 4, 204.

[11] Ross, R.M. and Watten, B.J. (1998) Importance of Rearing Unit Design and Stocking Density to the Behavior, Growth, and Metabolism of Lake Trout (Salvelinus namaycush). Aquacultural Engineering, 19, 41-56. 
https://doi.org/10.1016/S0144-8609(98)00041-7

[12] Tvinnereim, K. and Skybakmoen, S. (1989) Water Exchange and Self-Cleaning in Fish Rearing Tanks. In: De Pauw, N., Jaspers, E., Ackefors, H. and Wilkens, N., Eds., Aquaculture: A Biotechnology in Progress, European Aquaculture Society, Bredena, 1041-1047.

[13] Davidson, J. and Summerfelt, S. (2004) Solids Flushing, Mixing and Water Velocity Profiles within Large $\left(10\right.$ and $150 \mathrm{~m}^{3}$ ) Circular Cornell-Type Dual-Drain Tanks. Aquacultural Engineering, 32, 245-271. https://doi.org/10.1016/j.aquaeng.2004.03.009

[14] Plew, D.R., Klebert, P., Rosten, T.W., Aspaas, S. and Birkevold, J. (2015) Change to Flow and Turbulence Caused by Different Concentrations of Fish in a Circular Tank. Journal of Hydraulic Research, 53, 364-383. https://doi.org/10.1080/00221686.2015.1029016

[15] Näslund, J. and Johnsson, J.I. (2016) Environmental Enrichment for Fish in Captive Environments: Effects of Physical Structures and Substrates. Fish and Fisheries, 17, 1-30. https://doi.org/10.1111/faf.12088

[16] Berejikian, B.A., Tezak, E.P., Flagg, T.A., LaRae, A.L., Kummerow, E. and Mahnken, C.V.W. (2000) Social Dominance, Growth, and Habitat Use of Age-0 Steelhead Oncorhynchus mykiss Grown in Enriched and Conventional Hatchery Rearing Environments. Canadian Journal of Fisheries and Aquatic Sciences, 57, 628-636. https://doi.org/10.1139/f99-288

[17] Berejikian, B.A., Smith, R.J.F., Tezak, E.P., Schroder, S.L. and Knudsen, C.M. (1999) Chemical Alarm Signals and Complex Hatchery Rearing Habitats Affect Antipredator Behavior and Survival of Chinook Salmon (Oncorhynchus tshawytscha) Juveniles. Canadian Journal of Fisheries and Aquatic Sciences, 56, 830-838. https://doi.org/10.1139/f99-010

[18] Brown, C., Davidson, T. and Laland, K. (2003) Environmental Enrichment and Prior Experience of Live Prey Improve Foraging Behaviour in Hatchery-Reared Atlantic Salmon. Journal of Fish Biology, 63, 187-196. https://doi.org/10.1111/j.1095-8649.2003.00208.x

[19] Berejikian, B.A. and Tezak, E.P. (2005) Rearing in Enriched Hatchery Tanks Improves Dorsal Fin Quality of Juvenile Steelhead. North American Journal of Aquaculture, 67, 289-293. https://doi.org/10.1577/A05-002.1

[20] Brockmark, S., Neregård, L., Bohlin, T., Björnsson, B.T. and Johnsson, J.I. (2007) Effects of Rearing Density and Structural Complexity on Pre- and Postrelease Performance of Atlantic Salmon. Transactions of the American Fisheries Society, 136, 1453-1462. https://doi.org/10.1577/T06-245.1

[21] Fast, D.E., Neeley, D., Lind, D.T., Johnston, M.V., Strom, C.R., Bosch, W.J., Knudsen, C.M., Schroder, S.L. and Watson, B.D. (2008) Survival Comparison of Spring Chinook Salmon Reared in a Production Hatchery under Optimum Conventional and Seminatural Conditions. Transactions of the American Fisheries Society, 137, 1507-1518. https://doi.org/10.1577/T07-143.1

[22] Bergendahl, A.I., Miller, S., Depasquale, C., Giralico, L. and Braithwaite, V.A. (2017) Becoming a Better Swimmer: Structural Complexity Enhances Agility in Captive-Reared Fish. Journal of Fish Biology, 90, 1112-1117. https://doi.org/10.1111/jfb.13232

[23] Krebs, J., Crank, K.M., Krebs, E. and Barnes, M.E. (2017) Use of Bottom Structure and Tank Cover during Rainbow Trout Rearing in Circular Tanks. Journal of Fisheries and Livestock Production, 5, 3. 
[24] Baynes, S.M. and Howell, B.R. (1993) Observations on the Growth, Survival and Disease Resistance of Juvenile Common Sole, Soleasolea L. Aquaculture and Fisheries Management, 24, 95-100.

[25] Tuckey, L.M. and Smith, T.I. (2001) Effects of Photoperiod and Substrate on Larval Development and Substrate Preference of Juvenile Southern Flounder, Paralichthyslethostigma. Journal of Applied Aquaculture, 11, 1-20. https://doi.org/10.1300/J028v11n01_02

[26] Oca, J. and Masalo, I. (2007) Design Criteria for Rotating Flow Cells in Rectangular Aquaculture Tanks. Aquacultural Engineering, 36, 36-44. https://doi.org/10.1016/j.aquaeng.2006.06.001

[27] Kientz, J.L. and Barnes, M.E. (2016) Structural Complexity Improves the Rearing Performance of Rainbow Trout in Circular Tanks. North American Journal of Aquaculture, 78, 203-207. https://doi.org/10.1080/15222055.2016.1159629

[28] Kientz, J.L., Crank, K.M. and Barnes, M.E. (2018) Enrichment of Circular Tanks with Vertically Suspended Strings of Colored Balls Improves Rainbow Trout Rearing Performance. North American Journal of Aquaculture, 80, 162-167.

https://doi.org/10.1002/naaq.10017

[29] Crank, K.M., Kientz, J.L. and Barnes, M.E. (2018) An Evaluation of Vertically-Suspended Environmental Enrichment Structures during Rainbow Trout Oncorhynchus mykiss Rearing. North American Journal of Aquaculture, $\mathbf{8 0 .}$

[30] Krebs, E., Huysman, N., Voorhees, J.M. and Barnes, M.E. (2018) Suspended Arrays Improve Rainbow Trout Growth during Hatchery Rearing in Circular Tanks. International Journal of Aquaculture and Fishery Sciences, 4, 27-30.

[31] White, S.C., Krebs, E., Huysman, N., Voorhees, J.M. and Barnes, M.E. (2018) Use of Suspended Plastic Conduit Arrays during Brown Trout and Rainbow Trout Rearing in Circular Tanks. North American Journal of Aquaculture, $\mathbf{8 0 .}$

[32] Good, C., May, T., Crouse, C., Summerfelt, S. and Welch, T.J. (2016) Assessing the Impacts of Swimming Exercise and the Relative Susceptibility of Rainbow Trout Oncorhynchus mykiss (Walbaum) and Atlantic Salmon Salmo salar L. Following Injection Challenge with Weissellaceti. Journal of Fish Diseases, 39, 1387-1391. https://doi.org/10.1111/jfd.12468

[33] Liu, G., Wu, Y., Qin, X., Shi, X. and Wang, X. (2018) The Effect of Aerobic Exercise Training on Growth Performance, Innate Immune Response, and Disease Resistance in Juvenile Schizothoraxprenanti. Aquaculture, 486, 18-25. https://doi.org/10.1016/j.aquaculture.2017.12.006

[34] Waldrop, T., Summerfelt, S., Mazik, P. and Good, C. (2018) The Effects of Swimming Exercise and Dissolved Oxygen on Growth Performance, Fin Condition and Precocious Maturation of Early-Rearing Atlantic Salmon Salmo salar. Aquaculture Research, 49, 801-808. https://doi.org/10.1111/are.13511

[35] Sumida T., Kawahara, H., Shiotani, S., Sakakura, Y. and Hagiwara, A. (2013) Observations of Flow Patterns in a Model of a Marine Fish Larvae Rearing Tank. Aquacultural Engineering, 57, 24-31. https://doi.org/10.1016/j.aquaeng.2013.06.002

[36] Fausch, K.D. (1984) Profitable Stream Positions for Salmonids: Relating Specific Growth Rate to Net Energy Gain. Canadian Journal of Zoology, 62, 441-445. https://doi.org/10.1139/z84-067

[37] Kiessling, A., Higgs, D., Dosanjh, B. and Eales, J. (1994) Influence of Sustained Exercise at Two Ration Levels on Growth and Thyroid Function of All-Female Chinook Salmon Oncorhynchus tshawytscha in Seawater. Canadian Journal of Fisheries and Aquatic Sciences, 51, 1975-1984. https://doi.org/10.1139/f94-200 
[38] Buentello, J.A., Gatlin III, D.M. and Neill, W.H. (2000) Effects of Water Temperature and Dissolved Oxygen on Daily Feed Consumption, Feed Utilization and Growth of Channel Catfish (Ictalurus punctatus). Aquaculture, 182, 339-352. https://doi.org/10.1016/S0044-8486(99)00274-4

[39] Dabrowski, K., Lee, K.J., Guz, L., Verlhac, V. and Gabaudan, J. (2004) Effects of Dietary Ascorbic Acid on Oxygen Stress (Hypoxia or Hyperoxia), Growth and Tissue Vitamin Concentrations in Juvenile Rainbow Trout (Oncorhynchus mykiss). Aquaculture, 233, 383-392. https://doi.org/10.1016/j.aquaculture.2003.09.047 